\title{
Coping with interpersonal stress and psychological distress at work: comparison of hospital nursing staff and salespeople
}

This article was published in the following Dove Press journal:

Psychology Research and Behavior Management

I5 January 2014

Number of times this article has been viewed

\section{Tsukasa Kato \\ Department of Social Psychology, Toyo University, Tokyo, Japan}

Correspondence: Tsukasa Kato Department of Social Psychology, Toyo University, 5-28-20 Hakusan, Bunkyo-ku, Tokyo I I 2-8606, Japan $\mathrm{Tel}+8 \mathrm{I} 339454542$

Fax +8I 339457626

Email mtsukasa@hotmail.com

\begin{abstract}
Hospital nurses frequently experience relationships with patients as stressors in the workplace. Nurses' coping behavior is one potential buffering factor that can reduce the effects of job stress on their psychological functioning and well-being. In this study, the association between nurses' strategies for coping with interpersonal stress from patients and their psychological distress was examined. Participants included 204 hospital nurses and 142 salespeople, who were used as a comparison group. Participants completed measures of coping with interpersonal stress and psychological distress. Hospital nurses reported more psychological distress than did salespeople. Moreover, distancing coping was correlated with high psychological distress in both nurses and salespeople, and reassessing coping was correlated with low psychological distress in nurses. For nurses only, constructive coping appeared to be an effective strategy for reducing psychological distress. It is important for nurses to understand the role of constructive coping in nurse-patient communication and interaction.
\end{abstract}

Keywords: nurse, relationships with patients, interpersonal stress, coping behavior, job stress

\section{Introduction}

Previous studies have reported that nurses encounter multiple and ubiquitous stressors in the workplace, ${ }^{1-3}$ including work overload, shift patterns, lack of confidence in their efficacy as nurses, uncertainty about treatment, conflict with physicians/supervisors/ peers, and feelings of powerlessness. Such work-related stressors have a negative impact on the quality of care patients receive, as well as nurses' well-being, health, and work adaptation. ${ }^{2-7}$ For example, an analysis of data obtained from the Nurses' Health Study in the United States revealed that the relative risk for suicide among highly stressed female nurses compared to nurses with low work stress was 1.9 (95\% confidence interval [CI]: 0.8-4.7). ${ }^{8}$ In a review of studies on turnover in hospital nurses, Coomber and Barriball, in 2007, suggested that work-related stressors are associated with less job satisfaction, work shortage, higher rate of absence, and poor performance and efficiency. ${ }^{9}$ Thus, hospital nurses' experiences are important not only for patient care, but also for nurses themselves. Therefore, the present study evaluated how nurses coped with stress, particularly that originating from their relationships with patients, and the efficacy of their strategies in reducing subsequent psychological distress.

\section{Nurses' relationships with patients}

The present study focused on nurses' relationships with patients as a source of workrelated stress. Stress from interactions with patients is salient in nurses' lives. ${ }^{10-15}$ 
For example, Mariam et al found in 2012 that $60.8 \%$ of female nurses reported that quick-tempered patients were a primary source of work-related stress. ${ }^{16}$ Moreover, McGrath and Boore reported in 2003 that $27 \%$ of a sample of nurses in Northern Ireland reported that the emotional demands of patients were a source of moderate to high levels of stress. ${ }^{17}$ Indeed, interpersonal stressors originating in relationships with patients can have a strong effect on nurses' psychological distress, ${ }^{18,19}$ burnout, ${ }^{20,21}$ and job satisfaction. ${ }^{15,19}$ In addition, previous research has revealed that interpersonal stressors stemming from relationships with patients are related to emotional labor. ${ }^{22,23}$ Therefore, it is important for nursing researchers to investigate relationships with patients as a work-related stressor.

\section{Coping behavior}

According to the transactional theory proposed by Lazarus ${ }^{24}$ and Lazarus and Folkman, ${ }^{25}$ coping behavior is defined as "constantly changing cognitive and behavioral efforts to manage specific external and/or internal demands that are appraised as taxing or exceeding the resources of the person." ${ }^{24}$ This theory postulates that coping behavior affects well-being and adaptation, ${ }^{24}$ and the validity and utility of this hypothesis has been supported by numerous studies. ${ }^{24}$ For example, the National Institute for Occupational Safety and Health (NIOSH) job stress model suggests that coping behavior is one potential buffering factor that reduces or eliminates the effects of job stress on psychological and physiological homeostasis or stress-related illnesses. ${ }^{26}$ As a result, research on coping has received considerable attention in clinical research and practice. ${ }^{27}$

In addition, coping behavior has also received attention from nursing researchers. ${ }^{2,28,29}$ For example, in a study of female hospital nurses, ${ }^{30}$ it was reported that affectiveoriented coping was significantly associated with depressive symptoms, even after controlling for the effects of job stress, nursing-related work experience, and social support. Moreover, a study of hospital nurses in Australia and New Zealand found that type of coping strategy was significantly associated with physical and mental health, after controlling for the effects of job stress, years as a nurse in the current clinical unit, and income. ${ }^{31}$ Finally, Günüşen and Üstün also reported, in 2010, that burnout among nurses is reduced when coping skills training was provided. ${ }^{32}$

\section{Coping with interpersonal stressors}

Kato, in 2013, proposed three coping strategies for dealing with interpersonal stressors: distancing coping, reassessing coping, and constructive coping. ${ }^{33}$ Distancing coping refers to strategies that attempt to actively damage, disrupt, or dissolve a stressful relationship (eg, avoiding contact with the person, ignoring the person). It is possible that distancing coping leads to poor interpersonal relationships in the workplace. The deterioration of interpersonal relationships at work can also result in psychological and physiological dysfunctions. ${ }^{34}$ In samples of Japanese students, ${ }^{33}$ distancing coping was found to be positively and significantly correlated with psychological dysfunction, such as depressive symptoms, anxiety, and general psychological strain. On the other hand, reassessing coping incorporates efforts to patiently wait for an appropriate opportunity to act, such as a change or improvement in the situation (eg, taking a pragmatic view of the matter, deciding not to take the matter seriously). In samples of Japanese students, ${ }^{33}$ reassessing coping has been negatively and significantly correlated with psychological dysfunction. Finally, constructive coping involves actively seeking to improve, maintain, and/or sustain a relationship without aggravating the other individuals (eg, reflecting on one's own conduct, trying to understand the other person's feelings). This strategy emphasizes respecting others and maintaining harmonious relations. It is important for nurses to learn to cope with their relationships with patients as a work-related stressor, in order to decrease the negative effects on their well-being. To this author's knowledge, however, no study has examined the relationship between coping with interpersonal stressors and psychological dysfunction in nurses.

In the present study, the association between nurses' coping strategies used to deal with stress stemming from relationships with patients and resultant psychological distress were examined. In addition, the study aimed to uncover how nurses' coping responses compared with those in other industries with high levels of interpersonal stress; therefore, nurses' coping strategies and psychological distress were compared with those of department store salespeople. Previous research has shown that, as with nurses, salespeople are exposed to frequent potential interpersonal stressors in the workplace (eg, multiple interactions with customers), ${ }^{35,36}$ and has emphasized burnout and emotional exhaustion as important consequences of such stress. ${ }^{37,38}$ Therefore, salespeople were regarded as a suitable control group in the present study.

\section{Methods}

\section{Participants and procedures}

Two hundred and four hospital nurses and 142 salespeople participated in this study. All participants were 
Japanese and female. The mean age of the nursing sample was 29.89 years (range: 19-57 years; standard deviation [SD] $=9.28$ ), and the mean number of years working at hospitals was 9.12 (range 2-40 years). All salespeople were full-time department store workers, with a mean age of 34.20 years (range: $18-52$ years; $\mathrm{SD}=10.75$ ). Participants were recruited through short courses in stress management. The short course for nurses was hosted by a local nurses' association as job training. The short course for salespeople was hosted by the human resources department of the firm at which they were employed. Participation in the training was voluntary for both samples. The study survey was administered before the training. After signing an informed consent form, all participants completed a set of questionnaires in a group setting. All participants received a pen valued at 100 yen (approximately 1.25 USD) in exchange for completing each survey.

\section{Instruments}

All measures, originally written in English, were independently translated into Japanese by three native-speaking Japanese psychologists. They were then back-translated into English by a native English-speaking psychologist. After back-translation, the original and back-translated questionnaires were compared for discrepancies. The translated questionnaires were modified after a discussion between the translators.

\section{Coping with interpersonal stressors}

The Interpersonal Stress Coping Scale (ISCS) ${ }^{33}$ was used to measure coping with interpersonal stress. The ISCS consists of three subscales: distancing coping, reassessing coping, and constructive coping, each consisting of five items. In the present study, ISCS items were slightly modified for use with nurses and salespeople. For example, the item "tried to understand the other person's feelings" was modified to "tried to understand the patient's feelings" for the nurses, and to "tried to understand the shopper's feelings" for the salespeople. The participants were asked to rate the extent to which they used each item to deal with the patient or shopper on a 4-point Likert scale ranging from 0 (did not use) to 3 (used a great deal). The Cronbach's alphas for the nurse and salesperson samples in the present study were 0.795 and 0.734 for distancing coping, 0.842 and 0.780 for reassessing coping, and 0.770 and 0.738 for constructive coping, respectively.

\section{Psychological distress}

The 12-item General Health Questionnaire (GHQ12), ${ }^{39}$ designed as a general measure of health and psychopathology, used to measure psychological stress. The GHQ-12 is a self-report scale that has established reliability and validity. ${ }^{40}$ Participants were asked to report about their experiences within the past week on a 4-point Likert scale ranging from 0 (much less than usual) to 3 (better than usual). Cronbach's alphas in the present study were 0.904 and 0.878 for nurses and salespeople, respectively.

\section{Data analysis}

We provided three types of effect sizes: Cohen's $d$, Pearson's $r$, and Cohen's $q$. According to Cohen, ${ }^{41}$ small, medium, and large effect sizes correspond to $d$-values of $0.20,0.50$, and $0.80 ; R$-values of $0.10,0.30$, and 0.50 ; and $q$-values of $0.10,0.30$, and 0.50 , respectively. Cohen's $d$ is an effect size measure for $t$-values and Cohen's $q$ is an effect size measure for differences in correlation coefficients. All analyses were conducted with PASW Statistics (v 18.0.0; IBM Corporation, Armonk, NY, USA) and R software (v 3.0.1; The R Foundation for Statistical Computing, Vienna, Austria). Effect sizes and confidence intervals were calculated using the R software.

\section{Results}

\section{Between-sample differences in coping strategies and psychological distress}

Table 1 presents the means and SDs for coping strategies and psychological distress scores. Between-subject Student's $t$-tests were conducted to compare coping strategies and psychological distress scores in nurses and salespeople. The results revealed that the nurses reported higher psychological distress (mean $=9.44, \mathrm{SD}=6.43$ ) than the salespeople (mean $=7.84, \mathrm{SD}=7.49, t[344]=2.13, P<0.05,95 \% \mathrm{CI}:-3.08$ to $-0.12, d=0.23)$. There were no significant differences between the samples in terms of coping strategies; however, the mean scores for all coping strategies were higher for nurses than for salespeople $(P<0.10)$.

\section{Correlations between coping strategies and psychological distress}

The correlation coefficients between coping strategies and psychological distress are shown in Table 2. Distancing coping was significantly correlated with high psychological distress for nurses $(r[202]=0.235, P<0.001)$ and salespeople $(r[140]=0.207, P<0.05)$. Reassessing coping, however, was significantly correlated with low psychological distress for nurses $(r[202]=-0.224, P<0.001)$, but not for salespeople $(r[140]=-0.008)$. Finally, constructive coping was significantly correlated with low psychological 
Table I Means and standard deviations of coping strategies and psychological distress scores for nurse and salesperson samples

\begin{tabular}{|c|c|c|c|c|c|c|c|c|c|}
\hline \multirow[t]{2}{*}{ Variable } & \multicolumn{2}{|c|}{ Nurses $(\mathrm{N}=204)$} & \multicolumn{2}{|c|}{ Salespeople $(\mathrm{N}=\mid 42)$} & \multirow[t]{2}{*}{$t$-value } & \multirow[t]{2}{*}{$d f$} & \multicolumn{2}{|l|}{$95 \% \mathrm{Cl}$} & \multirow[t]{2}{*}{$d$-value } \\
\hline & Mean & SD & Mean & SD & & & $L L$ & UL & \\
\hline Psychological distress & 9.44 & 6.43 & 7.84 & 7.49 & $2.13 *$ & 344 & -3.08 & -0.12 & 0.23 \\
\hline \multicolumn{10}{|l|}{ Coping strategy } \\
\hline Distancing coping & 3.80 & 3.38 & 3.23 & 2.58 & $1.70 * *$ & 344 & -0.090 & 1.233 & 0.19 \\
\hline Reassessing coping & 7.75 & 3.68 & 7.12 & 3.09 & $1.66^{* *}$ & 344 & -0.116 & 1.366 & 0.18 \\
\hline Constructive coping & 6.29 & 2.84 & 5.77 & 2.94 & $1.65^{* *}$ & 344 & -0.100 & 1.139 & 0.18 \\
\hline
\end{tabular}

Notes: $95 \%$ confidence interval $(\mathrm{Cl})$ is for $t$-value. $d$-value (Cohen's $d$ ) is an effect size for $t$-value. $* P<0.05 ; * * P<0.10$.

Abbreviations: LL, lower limit; UL, upper limit; $d f$, degrees of freedom.

distress for nurses $(r[204]=-0.237, P<0.001)$, but not for salespeople $(r[140]=0.013)$.

\section{Differences between correlation coefficients}

Fisher's transformed $z$ scores were used to test for differences in the magnitude of the correlation coefficients (see Table 2). Results revealed that the correlation coefficient for the relationship between constructive coping and psychological distress was higher for nurses than for salespeople ( $z=2.31, P<0.05$, Cohen's $q=0.26$ ). There were no significant differences in correlation coefficients for relationships between distancing coping ( $z=0.27$, Cohen's $q=0.03$ ) or reassessing coping ( $z=1.38$, Cohen's $q=0.15$ ) and psychological distress.

\section{Discussion}

In the present study, coping strategies for dealing with workrelated stress stemming from relationships with patients was assessed in a sample of nurses, and compared with coping strategies and psychological distress in salespeople. Our findings revealed that nurses reported more psychological distress than did salespeople, which is consistent with previous studies that have reported that hospital nurses experience high levels of work-related stress. ${ }^{42}$ The nature of the interpersonal stressors, however, was different for nurses than for salespeople. For example, nurses often interact with the same patients over a longer period of time than do salespeople, who tend to have shorter encounters with shoppers.

Table 2 Correlations between coping strategies and psychological distress scores and testing the difference in correlation coefficients

\begin{tabular}{|c|c|c|c|c|}
\hline \multirow[t]{2}{*}{ Coping strategy } & $\begin{array}{l}\text { Nurses } \\
(\mathrm{N}=204)\end{array}$ & $\begin{array}{l}\text { Salespeople } \\
(N=142)\end{array}$ & z-value & q-value \\
\hline & $r$ & $r$ & & \\
\hline Distancing coping & $0.235 * * *$ & $0.207^{*}$ & 0.267 & 0.03 \\
\hline Reassessing coping & $-0.224 * * *$ & -0.075 & 1.384 & 0.15 \\
\hline Constructive coping & $-0.237 * * *$ & 0.013 & $2.308^{*}$ & 0.26 \\
\hline
\end{tabular}

Notes: $q$-value (Cohen's $q$ ) is an effect size for $z$-value. $* P<0.05$; $* * * P<0.00$ I.
In addition, the stressors that arise in nurse-patient relationships are complex (see the "Limitations and future research" subsection).

In addition, in the present study, the relationships between coping strategies and psychological distress were examined. The results indicated that distancing coping was correlated with high psychological distress, which is consistent with findings reported in a sample of college students. ${ }^{33}$ In addition, reassessing coping was correlated with less psychological distress, which is also consistent with the previous findings on college students. ${ }^{33}$

Interestingly, constructive coping was correlated with low psychological distress for nurses, but not for salespeople. Moreover, the correlation coefficient for the relationship between constructive coping and psychological distress was higher for nurses than for salespeople. Previous studies with college students also failed to demonstrate a significant relation between constructive coping and psychological dysfunction. Therefore, our findings indicate that, for nurses, constructive coping may be an effective strategy in reducing psychological distress. Constructive coping involves active efforts to improve, maintain, or sustain a relationship without aggravating other individuals involved. For nurses, it may be that relationships with patients are a potential source of stress; however, they are also a source of job satisfaction. ${ }^{43,44}$ Therefore, effective communication with patients enhances nurses' job satisfaction; consequently, it may reduce nurses' stress. Indeed, previous research has shown that nursepatient communication and interaction are essential aspects of nursing care..$^{45-49}$ The findings of the present study may help nurses better understand the importance of nurse-patient communication.

\section{Limitations and future research}

The results of the present study should be interpreted with caution, as this study presents several limitations. First, as the sample included only female Japanese nurses, our findings cannot be generalized to other populations (eg, Western populations). While several studies have indicated that nurses' 
perceptions of relationships with patients are sources of stress across cultures, ${ }^{13}$ other researchers found that coping with interpersonal stressors differs substantially between Asian and Western cultures. ${ }^{50,51}$ Thus, Japanese nurses' coping strategies for relationships with patients may differ from those in other populations.

Second, although the primary goal of coping research is to reveal how particular coping strategies affect psychological distress and well-being, ${ }^{24}$ such causality cannot be inferred from the present findings, as we utilized a cross-sectional design. Future studies should use longitudinal designs and laboratory paradigms in order to test whether nurses' coping strategies for relationships with patients predict psychological distress or well-being.

Finally, the unique nature of the nurse-patient relationships should be considered when interpreting the present findings. For the general population, interpersonal stressors include stressful episodes between two or more people that involve quarrels, arguments, negative attitudes or behavior, an uncomfortable atmosphere during a conversation or activity, and concern about hurting others' feelings. ${ }^{31}$ In addition to these basic interpersonal stressors, nurses' relationships with patients present stressors such as observing patients' suffering, ${ }^{12}$ death and dying, ${ }^{12,52,53}$ dealing with difficult or severely ill patients, ${ }^{11}$ and job expectations. ${ }^{54}$ Patient-related stressors are clearly complex; accordingly, caution is warranted in comparing nurses' relationship stressors with those of other workers. It could be useful for future nursing research to categorize relationships with patients according to the nature of the stressors.

\section{Conclusion}

We found that hospital nurses reported more psychological distress than did salespeople. Further, reassessing coping and constructive coping were effective in reducing psychological dysfunction for nurses, whereas distancing coping was not. It is important for nurses to understand the role of constructive coping in nurse-patient communication and interaction and to learn to adapt their coping strategies as needed.

\section{Acknowledgments}

This research was supported in part by Grant-in-Aid for Young Scientist 20730407 from the Ministry of Education, Culture, Sports, Science and Technology of Japan to Tsukasa Kato and by Grant-in-Aid for Japan Society for the Promotion of Science (JSPS) Fellows 00077 from JSPS to Tsukasa Kato.

\section{Disclosure}

The author reports no conflicts of interest in this work.

\section{References}

1. Clegg A. Occupational stress in nursing: a review of the literature. J Nurs Manag. 2001;9(2):101-106.

2. Lim J, Bogossian F, Ahern K. Stress and coping in Australian nurses: a systematic review. Int Nurs Rev. 2010;57(1):22-31.

3. McVicar A. Workplace stress in nursing: a literature review. $J A d v$ Nurs. 2003;44(6):633-642.

4. Hayes LJ, O'Brien-Pallas L, Duffield C, et al. Nurse turnover: a literature review. Int J Nurs Stud. 2006;43(2):237-263.

5. Irvine DM, Evans MG. Job satisfaction and turnover among nurses: integrating research findings across studies. Nurs Res. 1995;44(4): 246-253.

6. Lim J, Bogossian F, Ahern K. Stress and coping in Singaporean nurses: a literature review. Nurs Health Sci. 2010;12(2):251-258.

7. Yin JC, Yang KP. Nursing turnover in Taiwan: a meta-analysis of related factors. Int J Nurs Stud. 2002;39(6):573-581.

8. Feskanich D, Hastrup JL, Marshall JR, et al. Stress and suicide in the Nurses' Health Study. J Epidemiol Community Health. 2002;56(2): 95-98.

9. Coomber B, Barriball KL. Impact of job satisfaction components on intent to leave and turnover for hospital-based nurses: a review of the research literature. Int J Nurs Stud. 2007;44(2):297-314.

10. Aholaakko T. Reducing surgical nurses' aseptic practice-related stress. J Clin Nurs. 2011;20(23-24):3339-3350.

11. Dewe PJ. Identifying the causes of nurses' stress: a survey of New Zealand nurses. Work Stress. 1987;(1):15-24.

12. Florio GA, Donnelly JP, Zevon MA. The structure of work-related stress and coping among oncology nurses in high-stress medical settings: a transactional analysis. J Occup Health Psychol. 1998;3(3):227-242.

13. Glazer S, Gyurak A. Sources of occupational stress among nurses in five countries. Int J Intercult Relat. 2008;32(1):49-66.

14. Happell B, DwyerT, Reid-Searl K, Burke KJ, Caperchione CM, Gaskin CJ. Nurses and stress: recognizing causes and seeking solutions. J Nurs Manag. 2013;21(4):638-647.

15. Rout UR. Stress amongst district nurses: a preliminary investigation. J Clin Nurs. 2000;9(5):303-309.

16. Al-Otaibi M, Makhdom Y, Adel I. Sources of work stress and productivity among female health care workers in the emergency departments of general hospitals in Jeddah K.S.A. J Appl Med Sci. 2012;1:69-79.

17. McGrath A, Boore NR. Occupational stress in nursing. Int J Nurs Stud. 2003;40(5):555-565.

18. French SE, Lenton R, Walters V, Eyles J. An empirical evaluation of an expanded Nursing Stress Scale. J Nurs Meas. 2000;8(2):161-178.

19. Tyler P, Cushway D. Stress in nurses: the effects of coping and social support. Stress Med. 1995;11(1):243-251.

20. Shimizutani M, Odagiri Y, Ohya Y, et al. Relationship of nurse burnout with personality characteristics and coping behaviors. Ind Health. 2008;46(4):326-335.

21. Payne N. Occupational stressors and coping as determinants of burnout in female hospice nurses. J Adv Nurs. 2001;33(3):396-405.

22. Henderson A. Emotional labor and nursing: an under-appreciated aspect of caring work. Nurs Inq. 2001;8(2):130-138.

23. Mann S. A health-care model of emotional labour: an evaluation of the literature and development of a model. J Health Organ Manag. 2005;19(4-5):304-317.

24. Lazarus RS. Stress and Emotion: A New Synthesis. New York, NY: Springer; 1999.

25. Lazarus RS, Folkman S. Stress, Appraisal, and Coping. New York: Springer; 1984.

26. Hurrell JJ Jr, Kelloway EK. Psychological job stress. In Rom WN Markowitz SB, editors. Environmental and Occupational Medicine. 4th ed. Philadelphia, PA: Lippincott Williams and Wilkins; 2007:855-866. 
27. Kato T. Development of the Coping Flexibility Scale: evidence for the coping flexibility hypothesis. J Couns Psychol. 2012;59(2): 262-273.

28. Backer JH, Bakas T, Bennett SJ, Pierce PK. Coping with stress: programs of nursing research. In Rice VH, editor. Handbook of Stress, Coping, and Health: Implications for Nursing Research, Theory, and Practice. Thousand Oaks, CA: Sage Publications; 2000:223-263.

29. Shaw C. A framework for the study of coping, illness behaviour and outcomes. J Adv Nurs. 1999;29(5):1246-1255.

30. Lin H, Probst JC, Hsu Y. Depression among female psychiatric nurses in southern Taiwan: main and moderating effects of job stress, coping behaviour and social support. J Clin Nurs. 2010;19(15-16): 2342-2354.

31. Chang EM, Bidewell JW, Huntington AD, et al. A survey of role stress, coping and health in Australian and New Zealand hospital nurses. Int J Nurs Stud. 2007;44(8):1354-1362.

32. Günüşen NP, Üstün, B. An RCT of coping and support groups to reduce burnout among nurses. Int Nurs Rev. 2010;57(4):485-492.

33. Kato T. Assessing coping with interpersonal stress: development and validation of the Interpersonal Stress Coping Scale in Japan. Int Perspect Psychol. 2013;2(2):100-115.

34. Viswesvaran C, Sanchez JI, Fisher J. The role of social support in the process of work stress: a meta-analysis. J Vocat Behav. 1999;54(2): 314-334.

35. Cicala JE. The individual and organizational hazards of loneliness on salespeople. Review of Business and Finance Studies. 2014;5(1): 27-36.

36. Weeks WA, Fournier C. The impact of time congruity on salesperson's role stress: a person-job fit approach. Journal of Personal Selling and Sales Management. 2010;30(1):73-90.

37. Lewin JE, Sager JK. Salesperson burnout: a test of the copingmediational model of social support. Journal of Personal Selling and Sales Management. 2008;28(3):233-246.

38. Low GS, Cravens DW, Grant K, Moncrief WC. Antecedents and consequences of salesperson burnout. Eur J Mark. 2001;35(5/6):587-611.

39. Goldberg DP, Williams P. A User's Guide to the General Health Questionnaire. Windsor: NFER-Nelson; 1988.
40. Doi Y, Minowa M. Factor structure of the 12-item General Health Questionnaire in the Japanese general adult population. Psychiatry Clin Neurosci. 2003;57(4):379-383.

41. Cohen J. Statistical Power Analysis for the Behavioral Sciences. 2nd ed. Hillsdale, NJ: Erlbaum; 1988.

42. Duquette A, Kérouac S, Sandhu BK, Beaudet L. Factors related to nursing burnout: a review of empirical knowledge. Issues Ment Health Nurs. 1994;15(4):337-358.

43. Lynn MR, Morgan JC, Moore KA. Development and testing of the Satisfaction in Nursing Scale. Nurs Res. 2009;58(3):166-174.

44. McQueen A. Nurse-patient relationships and partnership in hospital care. J Clin Nurs. 2000;9(5):723-731.

45. Caris-Verhallen WM, Kerkstra A, Bensing JM. The role of communication in nursing care for elderly people: a review of the literature. J Adv Nurs. 1997;25(5):915-933.

46. Fleischer S, Berg A, Zimmermann M, Wüste K, Behrens J. Nurse-patient interaction and communication: a systematic literature review. J Public Health. 2009;17(5):339-353.

47. Hemsley B, Balandin S, Worrall L. Nursing the patient with complex communication needs: time as a barrier and a facilitator to successful communication in hospital. J Adv Nurs. 2012;68(1):116-126.

48. May C. Research on nurse-patient relationships: problems of theory, problems of practice. J Adv Nurs. 1990;15(3):307-315.

49. Shattell M. Nurse-patient interaction: a review of the literature. J Clin Nurs. 2004;13(6):714-722.

50. Cheng C, Wang F, Golden DL. Unpacking cultural differences in interpersonal flexibility: role of culture-related personality and situational factors. J Cross Cult Psychol. 2011;42(3):425-444.

51. Lam AG, Zane NWS. Ethnic differences in coping with interpersonal stressors: a test of self-construals as cultural mediators. J Cross Cult Psychol. 2004;35(4):446-459.

52. Gray-Toft P, Anderson JG. The Nursing Stress Scale: development of an instrument. J Behav Assess. 1981;3(1):11-23.

53. Harris PE. The Nurse Stress Index. Work Stress. 1989;3(4):335-346.

54. Billeter-Koponen S, Fredén L. Long-term stress, burnout and patientnurse relations: qualitative interview study about nurses' experiences. Scand J Caring Sci. 2005;19(1):20-27.
Psychology Research and Behavior Management

\section{Publish your work in this journal}

Psychology Research and Behavior Management is an international, peerreviewed, open access journal focusing on the science of psychology and its application in behavior management to develop improved outcomes in the clinical, educational, sports and business arenas. Specific topics covered include: Neuroscience, memory \& decision making; Behavior

\section{Dovepress}

modification \& management; Clinical applications; Business \& sports performance management; Social and developmental studies; Animal studies. The manuscript management system is completely online and includes a quick and fair peer-review system. Visit http://www.dovepress. $\mathrm{com} /$ testimonials.php to read real quotes from published authors. 